\title{
Valores normativos do desempenho motor: Construção de cartas percentílicas baseadas no método LMS de Cole \& Green
}

\author{
Motor performance reference values: Percentile charts based on Cole and \\ Green LMS method
}

\author{
Raquel Nichele de Chaves, ${ }^{1 *}$ Adam Baxter-Jones, ${ }^{2}$ José António Ribeiro Maia ${ }^{1}$ \\ ARTIGO ORIGINAL | ORIGINAL ARTICLE
}

\begin{abstract}
O propósito principal deste estudo foi construir valores de referência percentílica para provas de aptidão física (AptF), ao mesmo tempo que contrasta, graficamente, os níveis de AptF de crianças e adolescentes vouzelenses com os de outras pesquisas nacionais e internacionais. Foi avaliada uma amostra de 1920 crianças e adolescentes, com idades entre os 7 e os 17 anos em cinco testes motores: dinamometria manual, impulsão horizontal, corrida vaivém, corrida de 50 jardas e corrida/marcha da milha. As cartas percentílicas foram construídas com base no método LMS, implementado no software LMSchartmarker Pro versão 2.54. Os resultados sugerem forte variabilidade interindividual no desempenho motor. Em todas as provas, os rapazes apresentaram valores médios mais elevados do que as raparigas, sobretudo durante o período circum-pubertário. Na comparação do percentil 50 da prova de força manual, crianças e jovens vouzelenses tiveram desempenho similar aos de outros estudos, enquanto nas provas de impulsão horizontal, corrida vaivém e de 50 jardas constatou-se superioridade da amostra norte-americana. Na corrida/marcha da milha, a performance dos vouzelenses foi superior às amostras açoriana e americana.
\end{abstract}

Palavras-chave: aptidão física, cartas de referência, percentis, crianças, adolescentes

ABSTRACT

The main purpose of this study was to construct reference standards for physical fitness (PF) values, and to graphically highlight differences between Vouzela children and adolescents' PF levels with those from other national and international references samples. Sample comprised 1920 children and adolescents aged 7 to 17 years. PF was assessed by five tests: handgrip, standing jump, shuttle run, 50-yard dash and 1-mile walk/run. Centile charts were constructed using the LMS method using the LMSchartmarker Pro software. Results suggest pervasive interindividual differences in motor performance. Boys showed higher values than girls for all tests, mainly during the pubertal period. Vouzela children and adolescents had similar performance to other samples in handgrip test, while the 50th percentile values from US sample were higher in standing jump, shuttle run, 50-yard dash tests. Vouzela performance in one mile walk/run was better than Azores and US samples.

Keywords: physical fitness, reference charts, percentile, children, adolescents

Artigo recebido a 26.02.2013; $1^{\text {a }}$ Revisão 24.06.2013; Aceite 27.07.2013

${ }^{1}$ CIFI2D - Faculdade de Desporto, Universidade do Porto, Portugal

${ }^{2}$ College of Graduate Studies and Research University of Saskatchewan, Canadá

* Autor correspondente: Laboratório de Cineantropometria, Faculdade de Desporto da Universidade do Porto, Rua Dr. Plácido Costa, 91, 4200-450 Porto, Portugal; E-mail: raquelnichele@live.com.pt 


\section{INTRODUÇÃO}

A aptidão física (AptF) procura expressar, de forma integrada, um conjunto multivariado e parcimonioso de funções e estruturas corporais envolvidas na performance desportivomotora (Ardoy et al., 2011), usualmente designadas de componentes morfológica, muscular, motora, cardiorrespiratória e metabólica (Bouchard \& Shephard, 1994). Não obstante a pluralidade do seu conceito (Freitas, Marques, \& Maia, 1997), pode ser genericamente definida como o estado ou a condição que permite a execução de atividades físico-motoras que envolvem esforços físicos, sem que se instale fadiga excessiva (Malina, Bouchard, \& Bar-Or, 2004). Além disso, tem sido consensual classificar as suas componentes em dois domínios aparentemente distintos: (1) as que se associam ao desempenho atlético, necessários à eficiência desportiva, e (2) as que se associam ao estado de saúde, isto é, as que atuam na promoção da saúde (Guedes, 2007; Safrit, 1990).

Algumas das principais razões da pesquisa dos níveis de AptF associada à saúde de crianças e jovens, não atletas, centram-se no fato da infância e adolescência serem consideradas janelas críticas para o desenvolvimento de proficiência motora, além de serem, também, momentos sensíveis à influência de fatores ambientais que condicionam a estabilização de comportamentos saudáveis (Bustamante, Beunen, \& Maia, 2012; Freitas et al., 2002; Guedes \& Guedes, 1997; Ortega et al., 2011; Silva, Beunen, \& Maia, 2011), isto é, a participação efetiva em atividades desportivas e/ou da prática regular de exercício físico (Guedes, 2007). Estilos de vidas construídos e desenvolvidos nestes períodos, saudáveis ou não, poderão influenciar comportamentos e estados de saúde na fase adulta (Malina, 2001).

É compromisso do estado Português, consagrado pela Lei de Bases do Sistema Educativo nº46/86 e pela Organização da Estrutura Curricular do Ensino Básico e Secundário (Jacinto, Carvalho, Comédias, \& Mira, 2001; Ministério da Educação e da Ciência [MEC], 1991a, 1991b, 1998, 2004), a promoção e desenvolvimento físico-motor adequados à infância e à adolescência. Neste sentido, a Educação Física Escolar possui um valor educativo inquestionável em termos da promoção de atividades físicas e desportivas, pedagogicamente orientadas, para o desenvolvimento multilateral e harmonioso do aluno, tendo definido como um dos principais propósitos, em todos os blocos de ensino, elevar o nível funcional das capacidades motoras condicionais de modo específico a cada nível de escolaridade (Jacinto et al., 2001; MEC, 1991a, 1991b, 1998, 2004). Embora tais aspetos devam ser desenvolvidos, também, em parceria com a comunidade, sobretudo na iniciação à prática desportiva em clubes, é atribuído ao Professor de Educação Física a diligência de identificar os níveis de desempenho motor de seus alunos com avaliações periódicas, monitorizar a sua mudança ao longo dos anos letivos, bem como proporcionar oportunidades diferenciadas para elevar as capacidades motoras a nível funcional, ou seja, conceber e realizar atividades físico-motoras sintonizadas com as necessidades das crianças e/ou do adolescentes (Jacinto et al., 2001; MEC, 1991a, 1991b, 1998, 2004).

A Organização da Estrutura Curricular do Ensino Básico e Secundário (Jacinto et al., 2001; MEC, 1991a, 1991b, 1998, 2004) sugere a avaliação criterial da AptF relacionada com a saúde, isto é, o uso adequado dos resultados dos testes motores administrados para inferir sobre o desempenho nas capacidades motoras avaliadas de acordo com valores de corte préestabelecidos. Contudo, tais pontos de corte não provêm de estudos na população portuguesa, a que se adiciona o fato de não existir, atualmente, um mecanismo confiável à proposição desta classificação, sugerindo níveis mínimos necessários à redução de risco de doenças crónico-degenerativas (Guedes, 2007; Pate \& Daniels, 2013).

O estudo da variabilidade dos níveis de AptF, sobretudo relacionada com a saúde, tem procurado produzir valores de referência locais, ou seja, específicos de diferentes populações, 
contextos ambientais e condições socioculturais (Bustamante et al., 2012; Maia et al., 2007; Silva et al., 2011). A construção de cartas percentílicas locais (i.e., específicas da região) representa um capital informativo importante na gestão sistemática e eficiente do processo de ensino-aprendizagem, no âmbito escolar e desportivo, bem como no auxílio ao planeamento de estratégias e intervenções de órgãos institucionais, neste caso, os departamentos de desporto das Câmaras Municipais. É evidente que estes propósitos têm uma elevada generalização a qualquer região, indiferentemente do seu país de origem. Daqui que o principal propósito da presente pesquisa seja apresentar valores de referência percentílica para cinco provas de AptF, nomeadamente, dinamometria manual, impulsão horizontal, corrida vaivém, corrida de 50 jardas e corrida/marcha da milha. Do mesmo modo, contrastará, graficamente, e atribuirá significado aos valores das crianças e jovens vouzelenses relativamente ao desempenho apresentado noutros estudos desenvolvidos no país, precisamente no arquipélago dos
Açores (Maia et al., 2007), e no exterior, concretamente na Espanha (Serrano et al., 2009), no Brasil (Silva et al., 2011) e nos EUA (Maia \& Lopes, 2007; Safrit, 1990).

\section{MÉTODO}

\section{Participantes}

Os participantes desta pesquisa provêm do "Projeto Vouzela Ativo", um estudo auxológico e epidemiológico, com delineamento transversal, sobre crescimento somático, desenvolvimento e saúde da população escolar do Concelho de Vouzela, Distrito de Viseu. A amostra foi constituída por crianças e adolescentes das 20 escolas municipais do Concelho, com idades compreendidas entre os 7 e os 17 anos, avaliados no ano de 2010. Somente na avaliação da aptidão cardiorrespiratória a amostra foi ampliada por dois momentos distintos: 2008 e 2010.

A Tabela 1 mostra o número de indivíduos agrupados por idade e sexo, representando $\approx 70 \%$ das crianças e adolescentes da rede escolar pública de Vouzela.

Tabela 1

Distribuição amostral para cada teste de aptidão física em função da idade e sexo

\begin{tabular}{|c|c|c|c|c|c|c|c|c|c|c|c|c|c|c|c|}
\hline \multirow[t]{2}{*}{ Idade } & \multicolumn{3}{|c|}{$\begin{array}{c}\text { Dinamometria } \\
\text { manual }\end{array}$} & \multicolumn{3}{|c|}{$\begin{array}{l}\text { Impulsão } \\
\text { horizontal }\end{array}$} & \multicolumn{3}{|c|}{$\begin{array}{l}\text { Corrida } \\
\text { vaivém }\end{array}$} & \multicolumn{3}{|c|}{$\begin{array}{l}\text { Corrida de } \\
50 \text { jardas }\end{array}$} & \multicolumn{3}{|c|}{$\begin{array}{c}\text { Corrida/Marcha } \\
\text { da milha }\end{array}$} \\
\hline & 우 & $\hat{0}$ & Total & 우 & $\hat{0}$ & Total & o & $\hat{0}$ & Total & o & 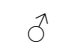 & Total & 우 & $\hat{0}$ & Total \\
\hline 7 & 45 & 48 & 93 & 44 & 49 & 93 & 44 & 47 & 91 & 38 & 41 & 79 & 81 & 78 & 159 \\
\hline 8 & 37 & 44 & 81 & 37 & 46 & 83 & 34 & 44 & 78 & 33 & 44 & 77 & 76 & 86 & 162 \\
\hline 9 & 48 & 46 & 94 & 48 & 46 & 94 & 47 & 46 & 93 & 44 & 42 & 86 & 89 & 81 & 170 \\
\hline 10 & 77 & 63 & 140 & 76 & 63 & 139 & 77 & 63 & 140 & 72 & 60 & 132 & 124 & 117 & 241 \\
\hline 11 & 57 & 55 & 112 & 55 & 52 & 107 & 55 & 51 & 106 & 54 & 51 & 105 & 106 & 119 & 225 \\
\hline 12 & 34 & 50 & 84 & 34 & 50 & 84 & 34 & 50 & 84 & 34 & 48 & 82 & 109 & 147 & 256 \\
\hline 13 & 47 & 57 & 104 & 46 & 58 & 104 & 45 & 57 & 102 & 42 & 57 & 99 & 43 & 56 & 99 \\
\hline 14 & 37 & 54 & 91 & 34 & 47 & 81 & 24 & 31 & 55 & 30 & 45 & 75 & 75 & 87 & 162 \\
\hline 15 & 35 & 31 & 66 & 28 & 17 & 45 & 21 & 13 & 34 & 27 & 17 & 44 & 78 & 62 & 140 \\
\hline 16 & 46 & 38 & 84 & 38 & 33 & 71 & 32 & 31 & 63 & 38 & 30 & 68 & 83 & 81 & 164 \\
\hline 17 & 42 & 25 & 67 & 35 & 17 & 52 & 32 & 11 & 43 & 31 & 17 & 48 & 80 & 62 & 142 \\
\hline Total & 505 & 511 & 1016 & 476 & 478 & 953 & 445 & 444 & 889 & 443 & 452 & 895 & 944 & 976 & 1920 \\
\hline
\end{tabular}




\section{Instrumentos e Procedimentos}

O projeto e os protocolos de avaliação foram aprovados pela Faculdade de Desporto da Universidade do Porto, pelos diretores dos agrupamentos escolares, e pelo Centro de Saúde de Vouzela. O consentimento livre e informado foi assinado pelos pais e/ou encarregados de educação dos alunos.

\section{Controlo da qualidade da informação}

O controlo da qualidade da informação passou por cinco etapas: (1) treino da equipe de avaliação por parte de avaliadores experientes (primeiro e último autores do presente artigo); (2) realização de retestes com uma amostra aleatória de 185 crianças e jovens num intervalo de duas semanas; (3) aplicação das provas sob a supervisão da primeira autora; (4) controlo da entrada da informação e análise exploratória prévia para identificar erros na entrada dos dados; (5) cálculo de estimativas de fiabilidade com base no coeficiente de correlação intraclasse $(R)$ e respetivos intervalos de confiança a 95\%: dinamometria manual, $R=0.973$ (IC95\% $=0.964-0.980$ ); impulsão horizontal, $R=0.933$ (IC95\% $=0.910-0.951$ ); corrida vaivém, $R=0.812$ (IC95\% $=0.748-0.860$ ); corrida de 50 jardas, $R=0.905$ (IC95\% $=0.823-$ 0.980); corrida/marcha da milha, $R=0.854$ $(\mathrm{IC} 95 \%=0.735-0.920)$.

\section{Avaliação da aptidão física}

Foi utilizado um conjunto de testes oriundos das baterias da AAHPER Youth Fitness Test (American Alliance for Health, Physical Education, and Recreation [AAHPER], 1976) e Fitnessgram (Welk \& Meredith, 2008) marcadores das seguintes componentes da AptF: agilidade e velocidade (corrida vaivém e corrida de 50 jardas), força explosiva dos membros inferiores (impulsão horizontal) e estática da mão (preensão), e capacidade aeróbia (corrida/ marcha da milha).

\section{Comparação com outros estudos}

A comparação dos níveis de AptF das crianças e adolescentes vouzelenses com resultados de pesquisas prévias foi efetuada com base nos valores medianos (P50). Deste modo, foram selecionados estudos de referência nacional e internacional que apresentam valores percentílicos das diferentes provas de AptF utilizadas nesta pesquisa: o Youth Fitness Test, publicado pela American Alliance for Health, Physical Education, Recreation and Dance (AAHPER, 1976), desenvolvido na população norte-americana; o estudo sobre crescimento e desempenho motor de crianças e jovens açorianos com idades entre os 6 e os 18 anos (Maia et al., 2007); o estudo realizado na região do Cariri, estado do Ceará, Brasil, designado "Crescer com Saúde no Cariri" (Silva et al., 2011); a pesquisa desenvolvida em Madrid, a qual é proveniente de um projeto maior, designado "Nutrição e Biodiversidade Humana" (Serrano et al., 2009).

\section{Análise Estatística}

Inicialmente foi efetuada a análise exploratória dos dados para detetar possíveis outliers, bem como analisar as distribuições das variáveis; de seguida foram calculadas a média, desvio-padrão e amplitude. A construção das cartas percentílicas foi efetuada, separadamente, para cada uma das provas de AptF em cada sexo. Os percentis foram obtidos pelo método LMS (Cole \& Green, 1992) implementado no software LMSchartmarker Pro versão 2.54 (Pan \& Cole, 2011). Para normalizar a distribuição dos valores em cada uma das variáveis, o método LMS recorre à transformação Box-Cox, específica para cada idade; os valores L, M e S são Cubic Splines em cada intervalo etário. Três curvas suavizadas e específicas de cada idade são produzidas, designadas de curva L (transformação Box-Cox), curva M (mediana) e curva $S$ (coeficiente de variação) com base na seguinte equação,

$$
C_{100 \alpha}(t)=M(t)\left[1+L(t) S(t) Z_{\alpha}\right]^{1 / L(t)}
$$

em que $Z_{\alpha}$ é o desvio normal equivalente para a amostra total, $\alpha$ e $C_{100 \alpha}(t)$ o percentil correspondente. A complexidade da suavização de 
cada curva é medida pelos graus de liberdade equivalentes para $L(t), M(t)$ e $S(t)$. Foram utilizados testes Q (Pan \& Cole, 2004; Royston \& Wright, 2000) para ajuizar da adequação do ajustamento, bem como das representações de Worm plots (Pan \& Cole, 2004; van Buuren \& Fredriks, 2001).

\section{RESULTADOS}

A Tabela 2 apresenta a distribuição dos scores $z$ das provas de AptF, nomeadamente, dinamometria manual, impulsão horizontal, corrida vaivém, corrida de 50 jardas e corri$\mathrm{da} /$ marcha da milha, para meninos e meninas. Esta distribuição foi comparada com as proporções esperadas de uma distribuição normal nos seguintes percentis: P3, P10, P25, P50, P75, P90, P97. Os resultados mostram elevada proximidade entre os valores esperados e os observados, sugerindo bom ajustamento das curvas percentílicas.

As curvas de referência para as provas de AptF das crianças e adolescentes vouzelenses, com idades compreendidas entre os 7 e os 17 anos e de ambos os sexos, são apresentadas nas Figuras 1 e 2.

Os valores percentílicos estão descritos nas Tabelas 3 e 4.

Em todas as provas os rapazes vouzelenses apresentaram valores mais elevados do que as meninas. Estas diferenças tendem a aumentar com a idade, sendo maiores durante o período

Tabela 2

Distribuição das frequências esperadas e observadas para meninas e meninos nos diferentes percentis de cada uma das provas de aptidão física, assumindo normalidade das distribuições

\begin{tabular}{|c|c|c|c|c|c|c|}
\hline \multicolumn{7}{|c|}{ Meninas } \\
\hline Percentil & $\begin{array}{c}\text { Esperado } \\
(\%)\end{array}$ & $\begin{array}{l}\text { Dinamometria } \\
\text { manual } \\
(\%)\end{array}$ & $\begin{array}{l}\text { Impulsão } \\
\text { horizontal } \\
\quad(\%)\end{array}$ & $\begin{array}{l}\text { Corrida } \\
\text { vaivém } \\
(\%)\end{array}$ & $\begin{array}{l}\text { Corrida de } 50 \\
\text { jardas } \\
(\%)\end{array}$ & $\begin{array}{c}\text { Corrida/ } \\
\text { Marcha da milha } \\
(\%)\end{array}$ \\
\hline 3 & 3 & 3.2 & 2.6 & 2.5 & 3.2 & 3.3 \\
\hline 10 & 7 & 6.8 & 8.2 & 8.0 & 7.1 & 8.2 \\
\hline 25 & 15 & 13.4 & 15.1 & 14.7 & 15.2 & 13.0 \\
\hline 50 & 25 & 27.6 & 23.7 & 25.0 & 22.3 & 26.4 \\
\hline 75 & 25 & 24.6 & 23.0 & 26.1 & 27.1 & 22.7 \\
\hline 90 & 15 & 13.6 & 17.0 & 11.9 & 16.3 & 15.6 \\
\hline 97 & 5 & 8.0 & 6.9 & 8.5 & 5.3 & 8.3 \\
\hline 100 & 2 & 2.8 & 3.7 & 3.2 & 3.4 & 2.5 \\
\hline \multicolumn{7}{|c|}{ Meninos } \\
\hline Percentil & $\begin{array}{c}\text { Esperado } \\
(\%)\end{array}$ & $\begin{array}{c}\text { Dinamometria } \\
\text { manual } \\
(\%)\end{array}$ & $\begin{array}{l}\text { Impulsão } \\
\text { horizontal } \\
(\%)\end{array}$ & $\begin{array}{c}\text { Corrida } \\
\text { vaivém } \\
(\%)\end{array}$ & $\begin{array}{l}\text { Corrida de } 50 \\
\text { jardas } \\
(\%)\end{array}$ & $\begin{array}{c}\text { Corrida/ } \\
\text { Marcha da milha } \\
(\%)\end{array}$ \\
\hline 3 & 3 & 2.6 & 2.6 & 2.8 & 3.6 & 2.5 \\
\hline 10 & 7 & 6.9 & 6.9 & 6.6 & 7.3 & 7.4 \\
\hline 25 & 15 & 15.7 & 15.8 & 15.2 & 11.6 & 16.6 \\
\hline 50 & 25 & 24.6 & 25.6 & 29.5 & 28.6 & 22.4 \\
\hline 75 & 25 & 25.0 & 22.2 & 19.8 & 24.6 & 24.8 \\
\hline 90 & 15 & 15.1 & 15.1 & 14.5 & 14.7 & 15.9 \\
\hline 97 & 5 & 8.3 & 8.8 & 8.5 & 5.4 & 7.1 \\
\hline 100 & 2 & 2.9 & 2.1 & 3.0 & 4.0 & 3.3 \\
\hline
\end{tabular}


Tabela 3

Valores percentílicos dos testes motores em meninas

\begin{tabular}{|c|c|c|c|c|c|c|c|}
\hline Idade & P3 & P10 & $\mathrm{P} 25$ & $\mathrm{P} 50$ & P75 & $\mathrm{P90}$ & P97 \\
\hline \multicolumn{8}{|c|}{ Dinamometria manual } \\
\hline 7 & 5.27 & 6.56 & 8.01 & 9.61 & 11.37 & 13.28 & 15.35 \\
\hline 8 & 6.70 & 8.24 & 9.94 & 11.80 & 13.82 & 16.00 & 18.34 \\
\hline 9 & 8.21 & 9.97 & 11.88 & 13.94 & 16.17 & 18.54 & 21.07 \\
\hline 10 & 9.82 & 11.75 & 13.84 & 16.08 & 18.47 & 21.00 & 23.68 \\
\hline 11 & 11.65 & 13.78 & 16.04 & 18.45 & 20.99 & 23.67 & 26.47 \\
\hline 12 & 13.54 & 15.84 & 18.26 & 20.80 & 23.46 & 26.23 & 29.10 \\
\hline 13 & 15.24 & 17.66 & 20.18 & 22.79 & 25.48 & 28.26 & 31.12 \\
\hline 14 & 16.65 & 19.15 & 21.71 & 24.34 & 27.02 & 29.77 & 32.56 \\
\hline 15 & 17.64 & 20.21 & 22.81 & 25.44 & 28.10 & 30.79 & 33.50 \\
\hline 16 & 18.18 & 20.83 & 23.47 & 26.10 & 28.73 & 31.36 & 33.98 \\
\hline 17 & 18.42 & 21.16 & 23.83 & 26.47 & 29.05 & 31.61 & 34.13 \\
\hline \multicolumn{8}{|c|}{ Impulsão horizontal } \\
\hline 7 & 69.30 & 78.10 & 87.52 & 97.57 & 108.26 & 119.60 & 131.60 \\
\hline 8 & 75.51 & 85.21 & 95.51 & 106.41 & 117.92 & 130.03 & 142.74 \\
\hline 9 & 81.63 & 92.24 & 103.42 & 115.15 & 127.43 & 140.25 & 153.60 \\
\hline 10 & 87.21 & 98.68 & 110.67 & 123.14 & 136.09 & 149.50 & 163.37 \\
\hline 11 & 92.76 & 105.11 & 117.89 & 131.10 & 144.69 & 158.67 & 173.02 \\
\hline 12 & 98.15 & 111.38 & 124.95 & 138.85 & 153.05 & 167.55 & 182.32 \\
\hline 13 & 102.66 & 116.65 & 130.89 & 145.34 & 160.01 & 174.87 & 189.91 \\
\hline 14 & 105.79 & 120.37 & 135.08 & 149.90 & 164.81 & 179.82 & 194.92 \\
\hline 15 & 107.72 & 122.74 & 137.75 & 152.75 & 167.74 & 182.73 & 197.71 \\
\hline 16 & 108.82 & 124.16 & 139.36 & 154.42 & 169.37 & 184.21 & 198.96 \\
\hline 17 & 109.70 & 125.34 & 140.68 & 155.78 & 170.65 & 185.32 & 199.82 \\
\hline \multicolumn{8}{|c|}{ Corrida vaivém } \\
\hline 7 & 17.42 & 15.96 & 14.81 & 13.88 & 13.11 & 12.45 & 11.89 \\
\hline 8 & 16.54 & 15.25 & 14.20 & 13.32 & 12.57 & 11.93 & 11.37 \\
\hline 9 & 15.71 & 14.57 & 13.60 & 12.78 & 12.06 & 11.42 & 10.86 \\
\hline 10 & 15.03 & 14.01 & 13.12 & 12.33 & 11.63 & 11.01 & 10.44 \\
\hline 11 & 14.49 & 13.56 & 12.73 & 11.97 & 11.29 & 10.67 & 10.10 \\
\hline 12 & 14.03 & 13.19 & 12.40 & 11.68 & 11.01 & 10.39 & 9.81 \\
\hline 13 & 13.65 & 12.88 & 12.14 & 11.44 & 10.78 & 10.16 & 9.56 \\
\hline 14 & 13.36 & 12.65 & 11.95 & 11.27 & 10.62 & 9.98 & 9.37 \\
\hline 15 & 13.16 & 12.49 & 11.82 & 11.16 & 10.51 & 9.86 & 9.22 \\
\hline 16 & 13.00 & 12.37 & 11.73 & 11.09 & 10.43 & 9.77 & 9.09 \\
\hline 17 & 12.86 & 12.26 & 11.65 & 11.02 & 10.37 & 9.69 & 8.97 \\
\hline \multicolumn{8}{|c|}{ Corrida de 50 jardas } \\
\hline 7 & 12.57 & 11.75 & 10.95 & 10.16 & 9.39 & 8.63 & 7.89 \\
\hline 8 & 12.13 & 11.32 & 10.55 & 9.80 & 9.09 & 8.41 & 7.75 \\
\hline 9 & 11.67 & 10.88 & 10.13 & 9.43 & 8.78 & 8.16 & 7.59 \\
\hline 10 & 11.24 & 10.46 & 9.74 & 9.09 & 8.48 & 7.93 & 7.42 \\
\hline 11 & 10.87 & 10.10 & 9.41 & 8.79 & 8.23 & 7.73 & 7.27 \\
\hline 12 & 10.56 & 9.80 & 9.13 & 8.55 & 8.03 & 7.57 & 7.16 \\
\hline 13 & 10.32 & 9.56 & 8.92 & 8.37 & 7.88 & 7.46 & 7.08 \\
\hline 14 & 10.14 & 9.38 & 8.76 & 8.23 & 7.78 & 7.39 & 7.04 \\
\hline 15 & 10.00 & 9.25 & 8.64 & 8.14 & 7.71 & 7.35 & 7.02 \\
\hline 16 & 9.90 & 9.15 & 8.56 & 8.08 & 7.68 & 7.33 & 7.03 \\
\hline 17 & 9.82 & 9.08 & 8.51 & 8.04 & 7.66 & 7.34 & 7.06 \\
\hline \multicolumn{8}{|c|}{ Corrida/Marcha da milha } \\
\hline 7 & 16.04 & 14.60 & 13.23 & 11.95 & 10.74 & 9.61 & 8.56 \\
\hline 8 & 15.77 & 14.21 & 12.76 & 11.43 & 10.20 & 9.07 & 8.03 \\
\hline 9 & 15.51 & 13.81 & 12.28 & 10.90 & 9.65 & 8.53 & 7.52 \\
\hline 10 & 15.06 & 13.24 & 11.64 & 10.23 & 8.99 & 7.90 & 6.94 \\
\hline 11 & 14.32 & 12.43 & 10.81 & 9.42 & 8.22 & 7.18 & 6.29 \\
\hline 12 & 13.68 & 11.78 & 10.17 & 8.80 & 7.64 & 6.65 & 5.79 \\
\hline 13 & 13.35 & 11.47 & 9.88 & 8.52 & 7.37 & 6.38 & 5.53 \\
\hline 14 & 13.23 & 11.41 & 9.83 & 8.48 & 7.31 & 6.30 & 5.43 \\
\hline 15 & 13.38 & 11.60 & 10.03 & 8.65 & 7.45 & 6.40 & 5.48 \\
\hline 16 & 13.67 & 11.91 & 10.34 & 8.94 & 7.69 & 6.58 & 5.60 \\
\hline 17 & 13.98 & 12.26 & 10.69 & 9.26 & 7.96 & 6.79 & 5.75 \\
\hline
\end{tabular}


66 | RN Chaves, A Baxter-Jones, JAR Maia

Tabela 4

Valores percentílicos dos testes motores em meninos

\begin{tabular}{|c|c|c|c|c|c|c|c|}
\hline Idade & P3 & $\mathrm{P} 10$ & P25 & $\mathrm{P} 50$ & P75 & P90 & P97 \\
\hline \multicolumn{8}{|c|}{ Dinamometria manual } \\
\hline 7 & 7.05 & 8.69 & 10.25 & 11.76 & 13.22 & 14.65 & 16.05 \\
\hline 8 & 8.26 & 10.09 & 11.90 & 13.69 & 15.45 & 17.20 & 18.94 \\
\hline 9 & 9.43 & 11.43 & 13.45 & 15.50 & 17.57 & 19.66 & 21.77 \\
\hline 10 & 10.89 & 13.03 & 15.28 & 17.62 & 20.05 & 22.56 & 25.16 \\
\hline 11 & 12.47 & 14.79 & 17.28 & 19.94 & 22.76 & 25.74 & 28.89 \\
\hline 12 & 14.33 & 16.93 & 19.75 & 22.80 & 26.07 & 29.57 & 33.29 \\
\hline 13 & 16.68 & 19.74 & 23.05 & 26.61 & 30.41 & 34.47 & 38.78 \\
\hline 14 & 19.39 & 23.11 & 27.07 & 31.23 & 35.60 & 40.17 & 44.93 \\
\hline 15 & 22.08 & 26.67 & 31.37 & 36.15 & 41.01 & 45.94 & 50.95 \\
\hline 16 & 24.36 & 29.93 & 35.36 & 40.66 & 45.87 & 51.00 & 56.05 \\
\hline 17 & 26.15 & 32.79 & 38.90 & 44.64 & 50.08 & 55.28 & 60.29 \\
\hline \multicolumn{8}{|c|}{ Impulsão horizontal } \\
\hline 7 & 81.97 & 90.79 & 100.03 & 109.67 & 119.71 & 130.15 & 140.99 \\
\hline 8 & 87.47 & 97.35 & 107.59 & 118.17 & 129.07 & 140.30 & 151.84 \\
\hline 9 & 92.95 & 103.95 & 115.22 & 126.72 & 138.47 & 150.43 & 162.61 \\
\hline 10 & 98.79 & 110.93 & 123.21 & 135.62 & 148.14 & 160.76 & 173.49 \\
\hline 11 & 104.93 & 118.27 & 131.57 & 144.85 & 158.12 & 171.36 & 184.58 \\
\hline 12 & 112.41 & 127.05 & 141.47 & 155.69 & 169.73 & 183.62 & 197.36 \\
\hline 13 & 121.21 & 137.15 & 152.62 & 167.71 & 182.46 & 196.91 & 211.09 \\
\hline 14 & 131.10 & 148.18 & 164.55 & 180.33 & 195.61 & 210.46 & 224.92 \\
\hline 15 & 140.68 & 158.53 & 175.43 & 191.57 & 207.07 & 222.01 & 236.47 \\
\hline 16 & 148.53 & 166.73 & 183.78 & 199.92 & 215.30 & 230.03 & 244.21 \\
\hline 17 & 154.98 & 173.28 & 190.26 & 206.20 & 221.30 & 235.67 & 249.43 \\
\hline \multicolumn{8}{|c|}{ Corrida vaivém } \\
\hline 7 & 16.52 & 15.13 & 14.06 & 13.19 & 12.47 & 11.86 & 11.34 \\
\hline 8 & 15.86 & 14.50 & 13.45 & 12.62 & 11.93 & 11.35 & 10.85 \\
\hline 9 & 15.18 & 13.87 & 12.87 & 12.06 & 11.41 & 10.85 & 10.38 \\
\hline 10 & 14.51 & 13.28 & 12.33 & 11.57 & 10.94 & 10.42 & 9.96 \\
\hline 11 & 13.85 & 12.75 & 11.88 & 11.17 & 10.57 & 10.06 & 9.62 \\
\hline 12 & 13.24 & 12.30 & 11.51 & 10.85 & 10.27 & 9.77 & 9.33 \\
\hline 13 & 12.71 & 11.91 & 11.21 & 10.59 & 10.04 & 9.54 & 9.09 \\
\hline 14 & 12.22 & 11.55 & 10.93 & 10.35 & 9.81 & 9.31 & 8.84 \\
\hline 15 & 11.78 & 11.21 & 10.65 & 10.11 & 9.59 & 9.08 & 8.59 \\
\hline 16 & 11.37 & 10.89 & 10.39 & 9.89 & 9.37 & 8.84 & 8.30 \\
\hline 17 & 11.00 & 10.59 & 10.14 & 9.67 & 9.16 & 8.60 & 7.98 \\
\hline \multicolumn{8}{|c|}{ Corrida de 50 jardas } \\
\hline 7 & 11.79 & 11.05 & 10.34 & 9.65 & 8.99 & 8.36 & 7.75 \\
\hline 8 & 11.52 & 10.76 & 10.04 & 9.36 & 8.72 & 8.12 & 7.55 \\
\hline 9 & 11.20 & 10.43 & 9.70 & 9.04 & 8.42 & 7.84 & 7.31 \\
\hline 10 & 10.85 & 10.06 & 9.34 & 8.69 & 8.09 & 7.55 & 7.05 \\
\hline 11 & 10.52 & 9.72 & 9.01 & 8.37 & 7.80 & 7.30 & 6.84 \\
\hline 12 & 10.23 & 9.42 & 8.72 & 8.11 & 7.57 & 7.09 & 6.67 \\
\hline 13 & 9.90 & 9.10 & 8.42 & 7.83 & 7.32 & 6.87 & 6.48 \\
\hline 14 & 9.50 & 8.71 & 8.06 & 7.50 & 7.03 & 6.61 & 6.25 \\
\hline 15 & 9.09 & 8.33 & 7.70 & 7.18 & 6.74 & 6.35 & 6.02 \\
\hline 16 & 8.75 & 8.01 & 7.41 & 6.92 & 6.51 & 6.16 & 5.85 \\
\hline 17 & 8.47 & 7.75 & 7.18 & 6.72 & 6.34 & 6.01 & 5.73 \\
\hline \multicolumn{8}{|c|}{ Corrida/Marcha da milha } \\
\hline 7 & 14.74 & 13.35 & 12.05 & 10.83 & 9.70 & 8.65 & 7.68 \\
\hline 8 & 14.59 & 13.06 & 11.67 & 10.40 & 9.24 & 8.20 & 7.25 \\
\hline 9 & 14.40 & 12.73 & 11.25 & 9.93 & 8.77 & 7.74 & 6.82 \\
\hline 10 & 13.93 & 12.16 & 10.63 & 9.31 & 8.17 & 7.18 & 6.33 \\
\hline 11 & 13.25 & 11.43 & 9.90 & 8.62 & 7.53 & 6.61 & 5.82 \\
\hline 12 & 12.65 & 10.82 & 9.32 & 8.07 & 7.04 & 6.17 & 5.43 \\
\hline 13 & 12.32 & 10.49 & 9.00 & 7.78 & 6.77 & 5.93 & 5.22 \\
\hline 14 & 12.23 & 10.39 & 8.90 & 7.69 & 6.69 & 5.85 & 5.15 \\
\hline 15 & 12.23 & 10.40 & 8.92 & 7.70 & 6.70 & 5.87 & 5.16 \\
\hline 16 & 12.38 & 10.54 & 9.05 & 7.84 & 6.83 & 5.99 & 5.29 \\
\hline 17 & 12.59 & 10.74 & 9.25 & 8.03 & 7.03 & 6.19 & 5.49 \\
\hline
\end{tabular}



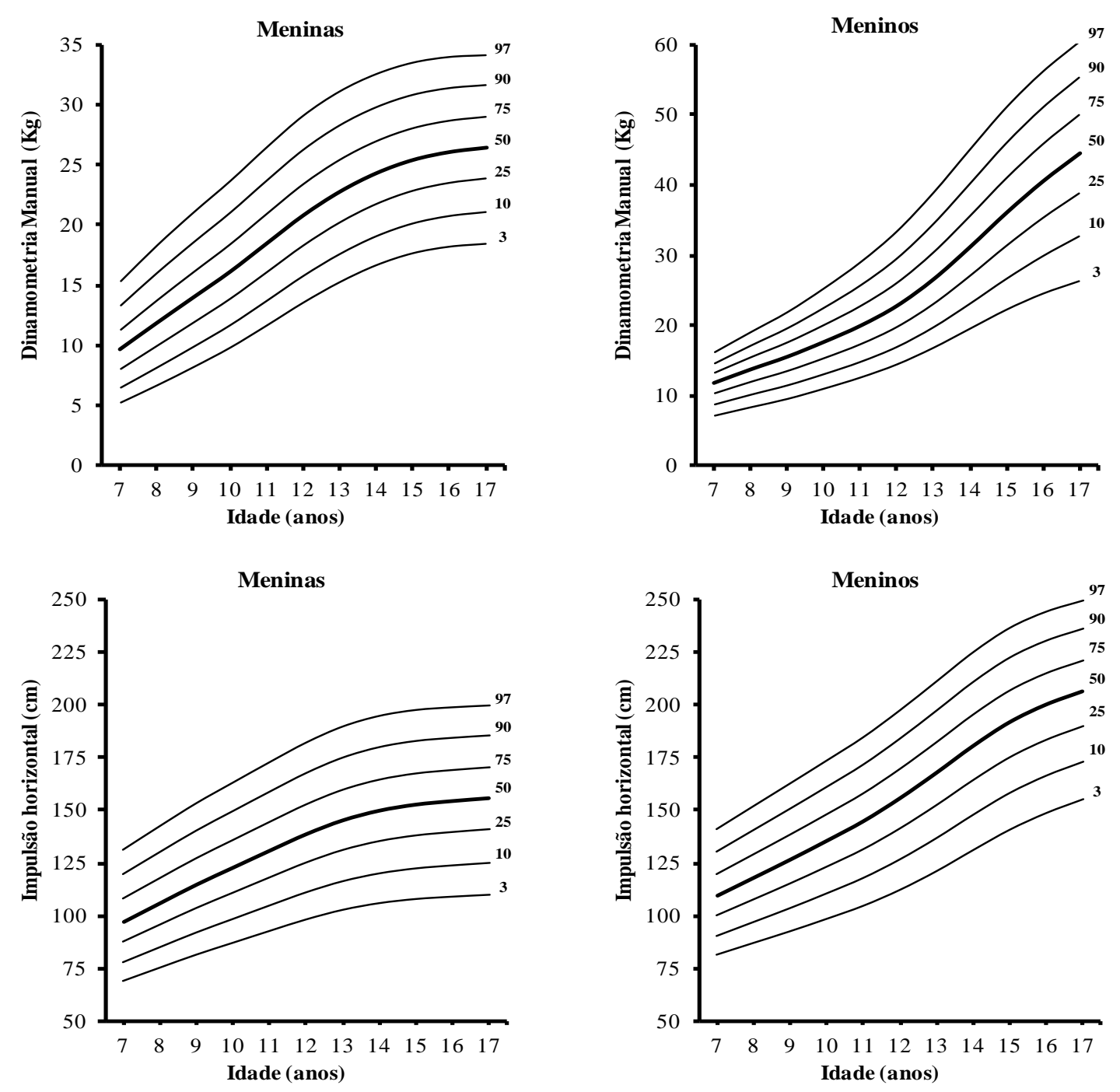

Figura 1. Cartas de referência percentílica dos testes de aptidão física: dinamometria manual e impulsão horizontal de meninas e meninos

circum-pubertário. Nas provas de força explosiva e estática, nomeadamente de preensão e impulsão horizontal, verifica-se aumento dos valores de força ao longo da idade. O comportamento das curvas percentílicas é distinto entre rapazes e raparigas, i.e., rapazes apresentam incrementos contínuos até os 17 anos, enquanto as raparigas tendem a estabilizar gradativamente e/ou aumentar ligeiramente os ganhos de força. $\mathrm{Na}$ prova de agilidade e de velocidade, os rapazes apresentam decréscimos de tempo de conclusão da prova ao longo das idades, sobretudo nas mais avançadas. As meninas reduzem este tempo, consideravel- mente, até os 12 anos de idade e, a partir daí, tendem a estabilizar. Na corrida/marcha da milha, o comportamento das curvas entre rapazes e raparigas são similares. Os rapazes têm melhores desempenhos em todas as idades e percentis, cujas diferenças se situam em torno dos $28 \mathrm{~s}$ a $1.52 \mathrm{~min}$. Constata-se que os rapazes e as raparigas reduzem o tempo de conclusão de prova até os 12 anos de idade e, a partir desta idade, tendem a manter e/ou diminuir o desempenho. Ressalta-se, ainda, que as raparigas, após os 13 anos de idade, têm maior aumento no tempo de realização desta prova. 

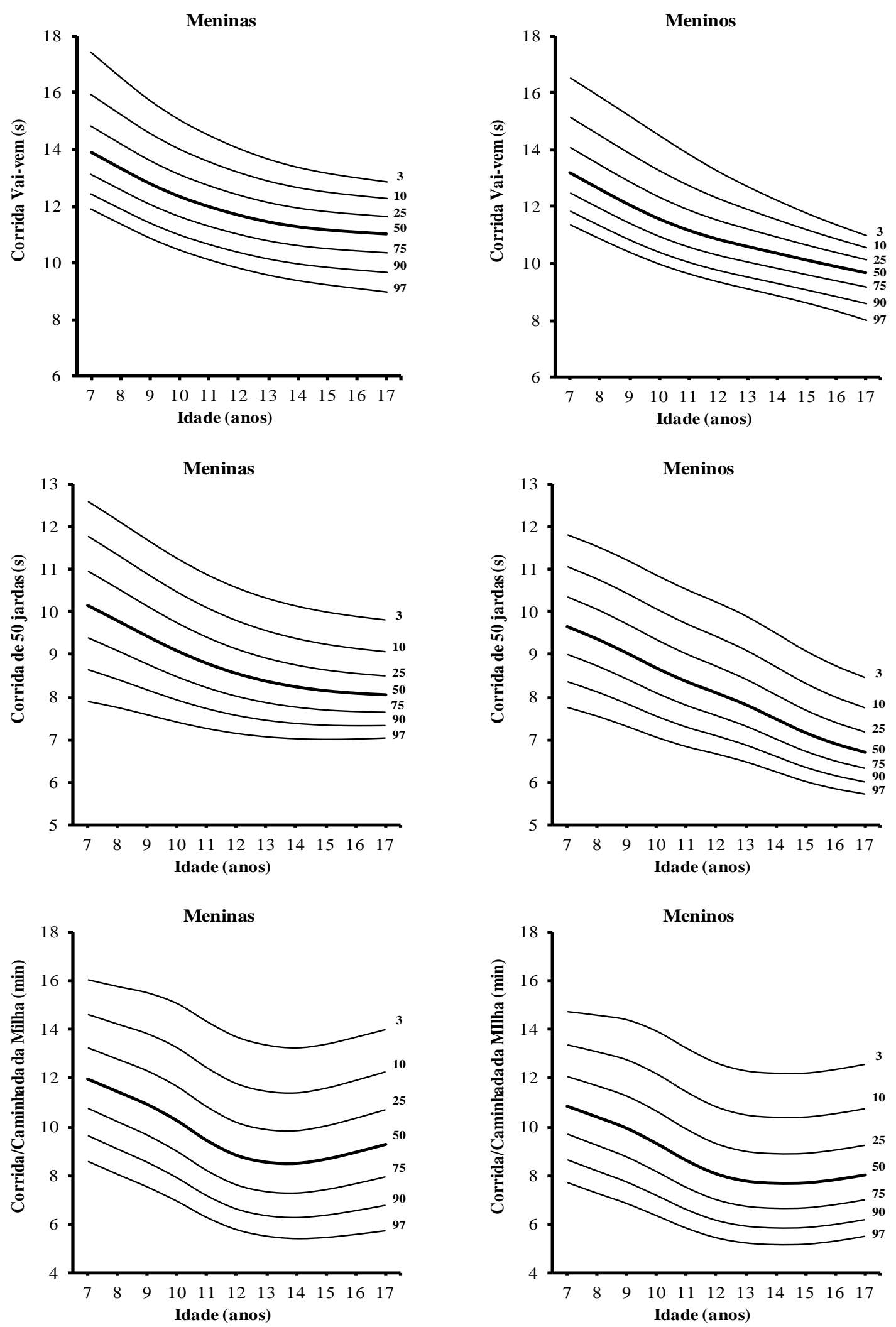

Figura 2. Cartas de referência percentílica dos testes de aptidão física: corrida vaivém, corrida de 50 jardas e corrida/marcha da milha de meninas e meninos 

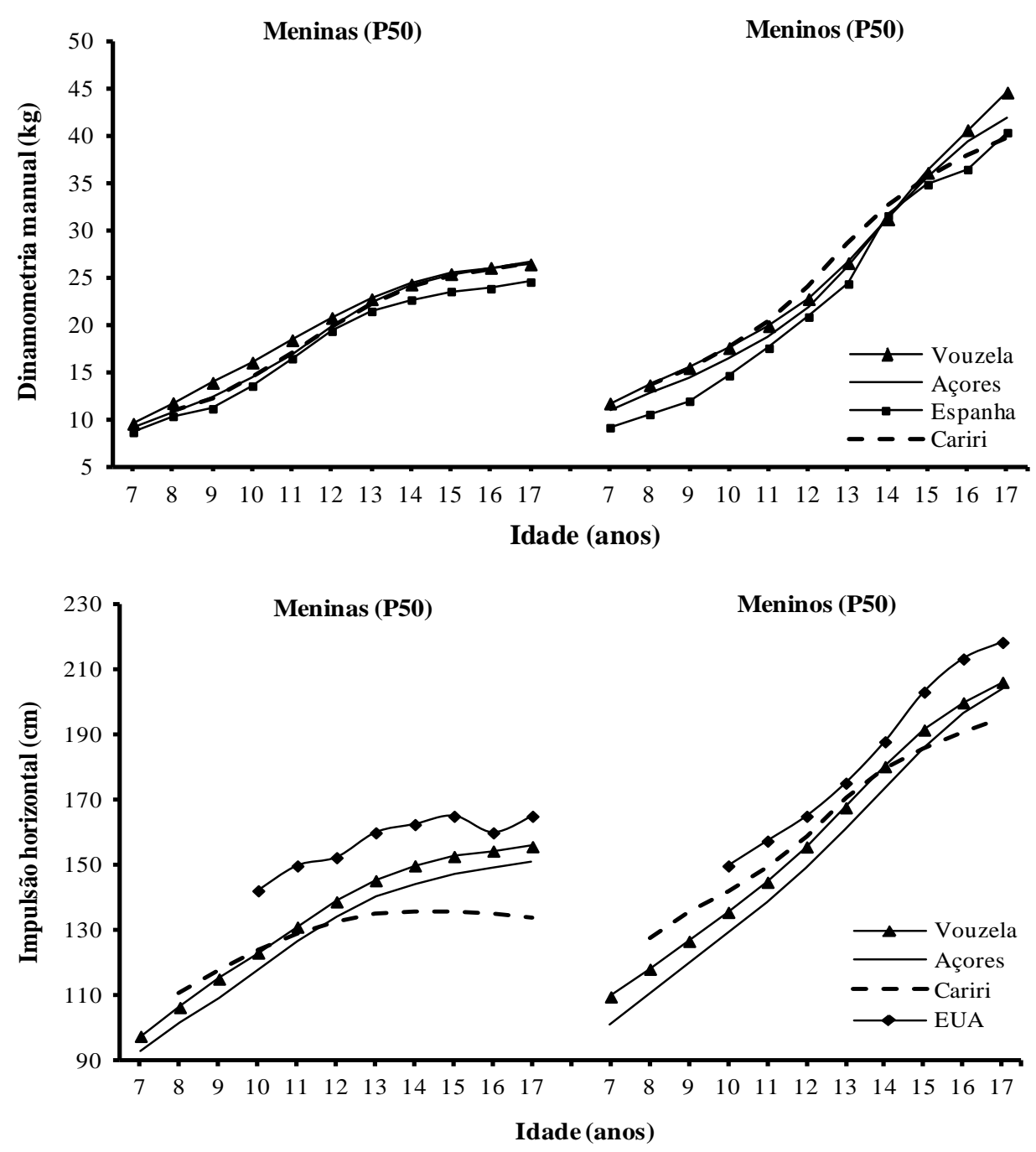

Figura 3. Comparação dos valores medianos (P50) da dinamometria manual e impulsão horizontal da população Vouzelense com outros estudos

As comparações dos valores medianos das crianças e adolescentes vouzelenses com outras referências, nacional e internacionais, nas provas de preensão e impulsão horizontal estão apresentadas na Figura 3. Na preensão, o comportamento da curva mediana é similar entre as amostras vouzelense, carirense, açoriana e espanhola, bem como os valores medianos em cada idade. A amostra espanhola apresenta menores valores neste teste. $\mathrm{Na}$ prova de impulsão horizontal, a amostra proveniente dos EUA tem valores superiores em ambos os sexos e um comportamento distinto nas rapa- rigas. Crianças e adolescentes vouzelenses reportam valores superiores aos açorianos em todas as idades; o mesmo não ocorre em comparação aos carirenses, os quais mostram valores mais elevados nas raparigas até os 10 anos e nos rapazes entre os 7 e os 14 anos de idade.

A Figura 4 ilustra as comparações dos valores medianos nas provas de corrida vaivém, corrida de 50 jardas e corrida/marcha da milha. $\mathrm{Na}$ corrida vaivém, os jovens norte-americanos apresentam melhores desempenhos, enquanto os vouzelenses realizam a corrida vaivém em menor tempo relativamente aos açorianos. As 

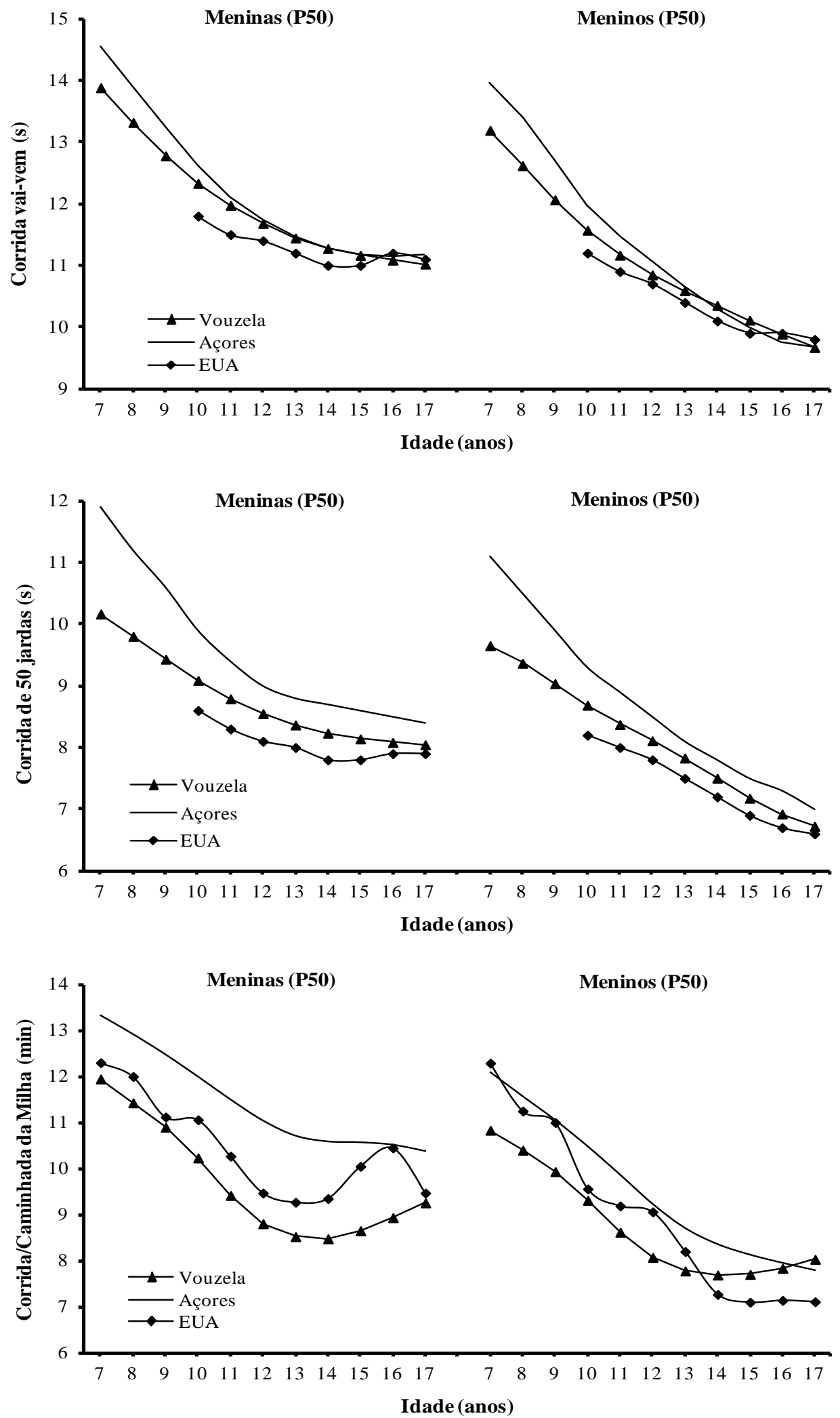

Figura 4. Comparação dos valores medianos (P50) da corrida vaivém, corrida de 50 jardas e corrida/caminhada da milha da população Vouzelense com outros estudos 
diferenças entre os valores medianos das três amostras reduzem-se ao longo da idade, em ambos os sexos, e a partir dos 15 anos a diferença é quase irrelevante. $\mathrm{Na}$ corrida de 50 jardas, rapazes e raparigas norte-americanos são os mais rápidos, e os vouzelenses têm melhores resultados que os açorianos. A amplitude das diferenças entre açorianos e vouzelenses mostra-se mais elevada até aos 10 anos de idade; as diferenças de desempenho entre vouzelenses e norte-americanos são insignificantes aos 17 anos, em ambos os sexos.

As crianças e adolescentes vouzelenses têm melhor resistência cardiorrespiratória que norte-americanos, exceto nos meninos a partir dos 14 anos, e nos seus pares açorianos. Ao contrário do que ocorre na maioria das provas anteriormente descritas, o comportamento das curvas medianas de cada amostra é distinto, sobretudo na amostra norte-americana. Os jovens vouzelenses e açorianos reduzem o tempo de realização de prova, de modo linear, até os 12 anos; a partir desta idade, há incrementos dos valores medianos nos vouzelenses, manutenção do tempo de prova nas raparigas açorianas e decréscimos sucessivos nos rapazes açorianos. Não se verifica linearidade na curva mediana dos jovens norte-americanos, mas sim oscilações consecutivas, incrementos e decréscimos, ao longo da idade.

\section{DISCUSSÃO}

Esta pesquisa foi realizada com o propósito de construir valores de referência percentílica para cinco provas de AptF, inspecionando o desempenho motor das crianças e jovens vouzelenses relativamente aos seus pares residentes em locais socioeconómica e geograficamente distintos, nomeadamente, Brasil, Espanha, EUA e Região Autónoma dos Açores. O uso de cartas percentílicas para descrever aspetos da trajetória modal e da variabilidade interindividual é uma das formas mais interessantes de apresentar o comportamento dos valores normativos em diferentes provas de AptF, face à sua relevância pedagógica e epidemiológica, sobretudo, quando são construídas com base em procedimentos metodológicos e analíticos robustos (Silva et al., 2011), assegurando o equilíbrio entre a fidedignidade dos resultados obtidos e a parcimónia estatística dos modelos produzidos. O método LMS apresenta estas características, sendo uma ferramenta estatística muito atual, vantajoso em comparação com outros métodos de estimação (Roelants, Hauspie, \& Hoppenbrouwers, 2009), com uma distinta representação gráfica e numérica dos perfis configuracionais do desempenho motor. A parcimónia no ajustamento dos modelos para cada prova e sexo é constatada com base nos resultados (não apresentados no texto) dos testes Q sugeridos por Royston e Wright (2000) e por Pan e Cole (2004), e na adequação da distribuição dos percentis, cujos resultados apontam diferenças muito pequenas entre os valores esperados e os observados em cada categoria percentílica, que se assemelham aos produzidos por Silva et al. (2011). Na fiabilidade do desempenho nas cinco provas de $\mathrm{AptF}$, os valores de correlação intraclasse apresentados foram elevados, entre 0.81 (corrida vaivém) e 0.97 (dinamometria manual), ressaltando a elevada consistência da performance motora de crianças e jovens vouzelenses, tal como reportado em outros estudos (Safrit, 1990; Silva et al, 2011).

Em geral, as curvas percentílicas construídas no presente estudo mostram uma forte variabilidade interindividual no desempenho das cinco provas utilizadas para avaliar a AptF de crianças e adolescentes vouzelenses, cujo perfil se assemelha ao reportado em outras regiões. Em consonância com a literatura prévia (Bustamante et al., 2012; Maia et al., 2007; Ortega et al., 2011; Silva et al., 2011), a expressão desta variabilidade é distinta entre os sexos e específica de cada prova, cujas trajetórias percentílicas estão condicionadas à idade, sendo notória a superioridade do desempenho dos rapazes em todas as provas de AptF. O comportamento distinto dos incrementos no desempenho das provas de força, velocidade e agilidade em rapazes e raparigas refletem uma interação complexa entre fatores biológicos e 
culturais associados à maturação biológica, diferenças de dimensões corporais, oportunidades e motivos para a prática de atividades desportivo-motoras (Malina et al., 2004).

A aptidão muscular refere-se, muito genericamente, à capacidade individual de gerar tensão contra uma resistência externa, resistir a repetidas contrações ou manter a contração máxima voluntária por um período de tempo prolongado e realizar uma contração máxima dinâmica (Ruiz et al., 2006). As provas de dinamometria manual e de impulsão horizontal têm sido amplamente aplicadas, sobretudo em estudos epidemiológicos, para a avaliação da força estática e força explosiva dos membros inferiores, respetivamente (Ortega, Ruiz, Castillo, \& Sjostrom, 2008). Os resultados encontrados na amostra vouzelense seguem um padrão semelhante ao referido em estudos prévios (Maia et al., 2007; Serrano et al., 2009; Silva et al., 2011) ou seja, o aumento linear da força ao longo da idade, cujas diferenças entre sexos são mais expressivas durante e após o período pubertário, face às mudanças na dinâmica muscular, sobretudo na maturação do tecido muscular, a qual ocorre diferentemente em meninos e meninas (Malina et al., 2004). Deste modo, os rapazes tendem a aumentar os seus níveis de força muscular, sobretudo após os 13 e 14 anos de idade, enquanto as meninas tendem a estabilizar seus ganhos e/ou apresentar ligeiros incrementos após esta idade.

Não obstante serem componentes associadas ao desempenho atlético (Guedes, 2007; Safrit, 1990), é sugerido que a agilidade e a velocidade, quando aliadas à melhoria dos níveis de aptidão muscular e cardiorrespiratória, podem apresentar efeitos positivos na saúde esquelética (Ortega et al., 2008). Embora haja uma ampla variedade de testes para avaliar tais capacidades e a necessidade de se obter mais informações a respeito da precisão, validade e fiabilidade dos resultados obtidos na aplicação destes testes (Ortega et al., 2008), é relativamente consensual que a corrida das 50 jardas e corrida vaivém, ambos provenientes da bateria de testes AAHPER (AAHPER, 1976;
AAHPERD, 1988) se mostram eficientes, de fácil aplicação e de resultados altamente fiáveis (Safrit, 1990). Os resultados da amostra vouzelense refletem um padrão de variação esperado e similar entre os dois testes, ou seja, rapazes e raparigas diminuem seu tempo de prova ao longo da idade, mas de modo distinto; os rapazes apresentam melhor desempenho, sobretudo após os 12/13 anos.

Em termos comparativos, nacional e internacionalmente, os valores medianos da dinamometria manual são muito similares. $\mathrm{Na}$ prova de impulsão horizontal, as diferenças são mais expressivas, sobretudo entre as meninas, salientando a superioridade de desempenho da amostra norte-americana. Nas corridas de vaivém e de 50 jardas, tal como mencionado anteriormente, a amostra dos EUA é mais veloz e ágil comparativamente aos seus pares açorianos e vouzelenses; nas idades mais avançadas (após os 15 anos), sobretudo na agilidade, os valores medianos tornam-se muito similares. Importa salientar que, para além de eventuais diferenças nos fatores biológicos, culturais e socioeconómicos, específicos de cada população, a que se associam fatores motivacionais relativos à prática desportiva, é provável que a desfasagem temporal, i.e., 32 anos entre o presente estudo e o estudo norte-americano, e aspetos estatísticos do cálculo dos percentis possam estar na origem da superioridade de valores medianos das crianças e jovens norteamericanos. É importante referir que os valores da amostra norte-americana provêm do Youth Fitness Test, publicado em 1976 pela American Alliance for Health, Physical Education, Recreation and Dance (AAHPER, 1976), cujos percentis foram calculados de modo empírico, i.e., sem recurso a um qualquer modo matemáticoestatístico.

Não obstante um esforço atual em reportar mudanças seculares no desempenho motor, e a presença de um certo conflito entre os resultados disponíveis (Smpokos, Linardakis, Papadaki, Lionis, \& Kafatos, 2012), há evidências de declínio dos valores médios de distintas componentes da AptF (Nishijima, Kokudo, \& 
Ohsawa, 2003; Tomkinson, 2007). Daqui que a superioridade dos resultados da amostra norteamericana possa estar condicionada à presença de uma tendência secular negativa no desempenho da força muscular explosiva dos membros inferiores, da agilidade e da velocidade de resultados mais atuais, sobretudo da amostra vouzelense.

A capacidade aeróbia tem sido uma das componentes mais estudadas no universo da AptF (Welk \& Meredith, 2008), seja na sua associação com a saúde, por estar associada à diminuição de diferentes fatores de risco de doenças cardiovasculares (Barlow et al., 2012; Blair et al., 1996; Grundy, Barlow, Farrell, Vega, \& Haskell, 2012), ou seja na sua relação com o desempenho atlético, já que níveis satisfatórios de aptidão cardiorrespiratória estão intimamente ligados à participação efetiva de crianças e jovens em inúmeras atividades físico-desportivas (Safrit, 1990). A corrida/marcha da milha tem sido amplamente utilizada para avaliar a resistência cardiorrespiratória de crianças e adolescentes, e em Portugal, é uma prova comummente aplicada nos diferentes níveis de ensino pelos professores de Educação Física (Jacinto et al., 2001; MEC, 1991a, 1991b, 1998, 2004). De acordo com Malina et al. (2004) é expetável uma melhoria da capacidade aeróbia ao longo da idade, sendo distinta entre os sexos, i.e., nos meninos verifica-se um aumento contínuo até aos 16 anos, enquanto as meninas tendem a aumentar sua capacidade aeróbia até aos 13 anos de idade. A partir destas idades, verifica-se a presença de um "plateau" de desempenho. Comportamentos similares são evidentes nas crianças e jovens açorianos. Não obstante terem melhor desempenho, os jovens vouzelenses têm um comportamento distinto - os rapazes estabilizam o seu desempenho por volta dos 12 anos e as meninas mostram um ligeiro aumento no tempo de prova, ou seja, uma diminuição dos níveis de capacidade aeróbia. Estas diferenças podem ser devidas a aspetos alométricos, precisamente pelo aumento corporal, muitas vezes não proporcional ao desenvolvimento dos órgãos do sistema cardiorrespiratório, e incrementos na quantidade massa gorda (Astrand \& Rodahl, 1986). Outro aspeto importante a ser ressaltado refere-se às condições socioeconómicas entre as amostras consideradas, sobretudo para explicar a melhor performance dos vouzelenses na corrida/marcha da milha. O Concelho de Vouzela preserva características peculiares de um contexto rural, divergindo dos grandes complexos urbanos, que influenciam a organização da rotina diária das crianças e dos jovens, fortemente condicionada pelo espaço disponível para o desenvolvimento de atividades lúdicas.

O presente estudo apresenta algumas limitações que importa referir. Em primeiro lugar há que mencionar a dimensão amostral e sua representatividade, sobretudo nas idades mais avançadas, embora sejam reportados estudos prévios de maior representatividade nacional na Suíça, cuja amostra foi de 662 crianças e adolescentes entre os 5 e os 18 anos de idade (Largo, Fischer, \& Rousson, 2003; McCarthy, Cole, Fry, Jebb, \& Prentice, 2006), i.e., efetivos semelhantes aos do presente estudo. Salientamos, também, que as estimativas das curvas $\mathrm{L}$, $\mathrm{M}$ e $\mathrm{S}$ dos resultados vouzelenses apresentam erros-padrão reduzidos, assegurando precisão no cálculo dos percentis. Em segundo lugar, a dificuldade em efetuar comparações mais objetivas entre diferentes populações repousa, necessariamente, em diferenças metodológicas na aplicação dos testes, bem como na obtenção dos valores percentílicos, a que se adicionam fatores condicionantes do desempenho, nomeadamente socioeconómicos, culturais e biológicos, para além dos efeitos da desfasagem temporal. Não obstante estes aspetos, o presente estudo possui alguns pontos fortes que passamos a referir: (1) a utilização de um método matemático-estatístico robusto, sofisticado e muito atual de estimação numérica e representação gráfica dos perfis configuracionais do desempenho motor das diferentes provas de AptF; (2) a aplicação rigorosa de testes com resultados altamente fiáveis da avaliação de diferentes componentes da AptF; (3) a 
apresentação de valores normativos do desempenho motor de crianças e jovens em idade escolar, de uma região de Portugal Continental; e (4) uma nova contribuição para ajuizar acerca do valor do planeamento e organização mais esclarecida e eficiente da prática educativa do Professor de Educação Física, sobretudo com a utilização de valores de referência mais próximos da realidade portuguesa, apresentando-se como um auxiliar importante em pesquisas sobre o crescimento e o desenvolvimento de crianças e jovens.

\section{CONCLUSÕES}

Em conclusão, os valores de referência construídos pelo presente estudo sugerem a forte variabilidade interindividual do desempenho motor nas diferentes provas de AptF, fato que fortalece a necessidade de estruturar a prática pedagógica de modo diversificado, criando oportunidades de vivências motoras capazes de atingir diferentes níveis de desempenho e, independentemente do grau de performance, garantir a melhoria da AptF de crianças e jovens. Relativamente às comparações dos valores medianos da prova de dinamometria manual, crianças e jovens vouzelenses tiveram desempenhos similares aos de outros estudos, enquanto nas provas de impulsão horizontal, corrida vaivém e de 50 jardas constatou-se superioridade da amostra norteamericana. Na corrida/marcha da milha, a performance dos vouzelenses foi superior às amostras açoriana e americana. Diferentes aspetos de natureza biológica, cultural e socioeconómica, específicos de cada população, a que se associam fatores motivacionais relativos à prática desportiva, bem como as características metodológicas e temporais de cada estudo, podem estar na origem das diferenças encontradas.

\section{Agradecimentos:}

Os autores agradecem à Câmara Municipal de Vouzela, ao Agrupamento de Escolas de Vouzela e a todos os profissionais da Educação Física e Desporto envolvidos no estudo; à Fundação para a Ciência e
Tecnologia (FCT) de Portugal (financiamento ao projeto de referência PTDC/DES/67569/2006) e à Coordenação de Aperfeiçoamento de Pessoal de Ensino Superior - CAPES (bolsa de Doutorado no exterior, processo de n. ${ }^{\circ}$ 623110-1). Um agradecimento especial a todas as crianças e adolescentes participantes do projeto "Vouzela Ativo". E queremos também expressar o nosso agradecimento aos revisores pelos comentários e sugestões que com certeza melhoraram o trabalho.

Conflito de Interesses:

Nada a declarar.

\section{Financiamento:}

Fundação para a Ciência e Tecnologia (FCT), Portugal (financiamento ao projeto de referência PTDC/DES/67569/2006); Coordenação de Aperfeiçoamento de Pessoal de Ensino Superior - CAPES, Ministério da Educação, Brasil (bolsa de Doutorado no exterior, processo n. ${ }^{\circ}$ 623110-1)

\section{REFERÊNCIAS}

American Alliance for Health, Physical Education, and Recreation [AAHPER] (1976). Youth Fitness Test Manual. Washington, DC: AAHPER.

American Alliance for Health, Physical Education, Recreation and Dance [AAHPERD] (1988). Physical best: A physical fitness education and assessment program best. Reston, VA: AAHPERD.

Ardoy, D.N., Fernandez-Rodriguez, J.M., Ruiz, J.R., Chillon, P., Espana-Romero, V., Castillo, M.J., \& Ortega, F.B. (2011). Improving physical fitness in adolescents through a school-based intervention: The EDUFIT study. Revista Española de Cardiologia, 64(6), 484-491. doi: 10.1016/j. recesp.2011.01.009

Astrand, P. O., \& Rodahl, K. (1986). Textbook of work physiology ( $3^{\mathrm{a}}$ ed). New York: McGrawHill.

Barlow, C.E., Defina, L.F., Radford, N.B., Berry, J.D., Cooper, K.H., Haskell, W.L., ... Lakoski, S.G. (2012). Cardiorespiratory fitness and long-term survival in "low-risk" adults. Journal of the American Heart Association, 1(4), e001354. doi: 10.1161/JAHA.112.001354

Blair, S.N., Kampert, J.B., Kohl, H.W., Barlow, C.E., Macera, C.A., Paffenbarger, R.S., Jr., \& Gibbons, L.W. (1996). Influences of cardiorespiratory fitness and other precursors on cardiovas- 
cular disease and all-cause mortality in men and women. Journal of the American Medical Association, 276(3), 205-210.

Bouchard, C., \& Shephard, R. (1994). Physical activity, fitness, and health: The model and key concepts. In C. Bouchard, R. Shephard \& T. Stephens (Eds.), Physical activity, fitness, and health International Proceedings and Consensus Statement (pp. 77-88). Champaign, Il: Human Kinetics.

Bustamante, A., Beunen, G., \& Maia, J. A. (2012). Valoración de la aptitud física en niños y adolescentes: Construcción de cartas percentílicas para la región central del Perú. Revista Peruana de Medicina Experimental y Salud Pública, 29(2), 188-197.

Cole, T. J., \& Green, P. J. (1992). Smoothing reference centile curves: The LMS method and penalized likelihood. Statistics in Medicine, 11(10), 1305-1319.

Freitas, D., Maia, J.A.R., Beunen, G., Lefevre, J., Claessens, A., Marques, A., ... Crespo, M. (2002). Crescimento somático, maturação biológica, aptidão física, actividade física e estatuto sócioeconómico de crianças e adolescentes madeirenses - $O$ Estudo de Crescimento da Madeira. Funchal: Universidade da Madeira.

Freitas, D., Marques, A., \& Maia, J.A.R. (1997). Aptidão Física da População Escolar da Região Autónoma da Madeira. Funchal: Universidade da Madeira.

Grundy, S.M., Barlow, C.E., Farrell, S.W., Vega, G.L., \& Haskell, W.L. (2012). Cardiorespiratory fitness and metabolic risk. American Journal of Cardiology, 109(7), 988-993. doi: 10.1016/j. amjcard.2011.11.031

Guedes, D.P. (2007). Implicações associadas ao acompanhamento do desempenho motor de crianças e adolescentes. Revista Brasileira de Educação Física e Esporte, 21, 37-60.

Guedes, D.P., \& Guedes, J.E. (1997). Crescimento, composição corporal e desempenho motor de crianças e adolescentes. São Paulo, SP: CLR Balieiro.

Jacinto, J., Carvalho, L., Comédias, J., \& Mira, J. (2001). Programa de Educação Física $\left(10^{\circ}, 11^{\circ}\right.$ e $12^{\circ}$ anos - Cursos Científico-Humanísticos e Cursos Tecnológicos). Lisboa: Ministério da Educação e da Ciência.

Largo, R.H., Fischer, J.E., \& Rousson, V. (2003). Neuromotor development from kindergarten age to adolescence: Developmental course and variability. Swiss Medical Weekly, 133, 193-199.
Maia, J.A.R., Lopes, V.P., Bustamante, A., Santos, M.L., Bacalhau, F., Silva, R.G., ... Prista, A. (2007). Crescimento e desempenho motor de crianças e jovens Açorianos: Cartas de referência para uso em Educação Física, Desporto, Pediatria e Nutrição. RAA; Porto: DRD/RAA - FADE/UP.

Maia, J.A.R., \& Lopes, V.P. (2007). Crescimento e desenvolvimento de crianças e jovens Açorianos: O que pais, professores, pediatras e nutricionistas gostariam de saber. RAA; Porto: DRD/RAA - FADE/UP.

Malina, R.M. (2001). Physical activity and fitness: pathways from childhood to adulthood. American Journal of Human Biology, 13(2), 162-172. doi: $10.1002 / 1520-6300(200102 / 03) 13: 2<162$ ::AID-AJHB1025>3.0.CO;2-T

Malina, R., Bouchard, C., \& Bar-Or, O. (2004). Growth, maturation and physical activity. Champaign, Il: Human Kinetics.

McCarthy, H.D., Cole, T.J., Fry, T., Jebb, S.A., \& Prentice, A.M. (2006). Body fat reference curves for children. International Journal of Obesity, 30(4), 598-602. doi: 10.1038/sj.ijo.0803232

Ministério da Educação e da Ciência [MEC] (1991a). Organização Curricular e Programas (3. ${ }^{\circ}$ ciclo do ensino básico). Lisboa: ME-DGEBS.

Ministério da Educação e da Ciência [MEC] (1991b). Organização Curricular e Programas $\left(2 .^{\circ}\right.$ ciclo do ensino básico) (Vol. I). Lisboa: MEDGEBS.

Ministério da Educação e da Ciência [MEC] (1998). Programa Educação Física. Plano de Organização de Ensino-Aprendizagem (2. ${ }^{\circ}$ ciclo do ensino básico) (Vol. II). Lisboa: ME-DGEBS.

Ministério da Educação e da Ciência [MEC] (2004). Organização Curricular e Programas - Ensino Básico: $1^{o}$ Ciclo (4 ed.). Algueirão-Mem Martins: Departamento da Educação Básica.

Nishijima, T., Kokudo, S., \& Ohsawa, S. (2003). Changes over the years in physical and motor ability in Japanese youth in 1964-97. International Journal of Sport and Health Science, 1, 164170.

Ortega, F.B., Artero, E.G., Ruiz, J.R., EspanaRomero, V., Jimenez-Pavon, D., VicenteRodriguez, G., ... Castillo, M.J. (2011). Physical fitness levels among European adolescents: The HELENA study. British Journal of Sports Medicine, 45(1), 20-29. doi: 10.1136/bjsm.2009.062679

Ortega, F.B., Ruiz, J.R., Castillo, M.J., \& Sjostrom, M. (2008). Physical fitness in childhood and adolescence: A powerful marker of health. In- 
ternational Journal of Obesity, 32(1), 1-11. doi: 10.1038/sj.ijo.0803774

Pan, H., \& Cole, T.J. (2004). A comparison of goodness of fit tests for age-related reference ranges. Statistics in Medicine, 23(11), 1749-1765.

Pan, H., \& Cole, T. J. (2011). LMS ChartMaker, a program to construct growth references using the LMS method. Version 2.54. Retrieved from http:// www.healthforallchildren.co.uk/

Pate, R.R., \& Daniels, S. (2013). Institute of Medicine Report on Fitness Measures and Health Outcomes in Youth. JAMA Pediatrics, 167, 221222. doi: 10.1001/jamapediatrics.2013.1464

Roelants, M., Hauspie, R., \& Hoppenbrouwers, K. (2009). References for growth and pubertal development from birth to 21 years in Flanders, Belgium. Annals of Human Biology, 36(6), 680694. doi: 10.3109/03014460903049074

Royston, P., \& Wright, E. M. (2000). Goodness-offit statistics for age-specific reference intervals. Statistics in Medicine, 19(21), 2943-2962.

Ruiz, J., Ortega, F., Gutierrez, A., Meusel, D., Sjöström, M., \& Castillo, M. (2006). Healthrelated fitness assessment in childhood and adolescence: A European approach based on the AVENA, EYHS and HELENA studies. Journal of Public Health, 14(5), 269-277. doi: 10.1007/s10 389-006-0059-z

Safrit, M. (1990). Introduction to Measurement in Physical Education and Exercise Science. St. Louis, Missouri: Times Mirror/Mosby College Publishing.
Serrano, M.D., Collazos, J.F., Romero, S.M., Santurino, M.S., Armesilla, M.D., del Cerro, J. L., \& de Espinosa, M.G. (2009). Handgrip strength in children and teenagers aged from 6 to 18 years: Reference values and relationship with size and body composition. Anales De Pediatria, 70, 340-348. doi: 10.1016/j.anpedi.2008.11.025

Silva, S., Beunen, G., \& Maia, J. (2011). Valores normativos do desempenho motor de crianças e adolescentes: O estudo longitudinal-misto do Cariri. Revista Brasileira de Educação Física e Esporte, 25, 111-125.

Smpokos, E.A., Linardakis, M., Papadaki, A., Lionis, C., \& Kafatos, A. (2012). Secular trends in fitness, moderate-to-vigorous physical activity, and TV-viewing among first grade school children of Crete, Greece between 1992/93 and 2006/07. Journal of Science and Medicine in Sport, 15, 129-135. doi: 10.1016/j.jsams.2011.08.006

Tomkinson, G. R. (2007). Global changes in anaerobic fitness test performance of children and adolescents (1958-2003). Scandinavian Journal of Medicine and Science in Sports, 17(5), 497-507. doi: 10.1111/j.1600-0838.2006.00569.x

van Buuren, S., \& Fredriks, M. (2001). Worm plot: A simple diagnostic device for modelling growth reference curves. Statistics in Medicine, 20(8), 1259-1277. doi: 10.1002/sim.746

Welk, G. J., \& Meredith, M. D. (2008). Fitnessgram/ Activitygram Reference Guide. Dallas, TX: The Cooper Institute.

Todo o conteúdo da revista Motricidade está licenciado sob a Creative Commons, exceto quando especificado em contrário e nos conteúdos retirados de outras fontes bibliográficas. 